\title{
Unreifes Olivenöl lindert Osteoarthrose-Symptome
}

Das Fruchtöl des Olivenbaums (Olea europea) enthält als Hauptbestandteil Triglyzeride mit einfach ungesättigten Fettsäuren, daneben auch einen unverseifbaren Anteil von 1-2\%. Dieser besteht aus Phytosterolen, Triterpenderivaten, Tocopherol und anderen Verbindungen. Gerade diese unverseifbare Fraktion scheint Komponenten zu enthalten, die für die entzündungshemmenden Eigenschaften des Olivenöls verantwortlich sind. Allerdings variiert die Zusammensetzung sowohl quantitativ als auch qualitativ erheblich während des Reifeprozesses der Frucht mit entsprechend unterschiedlicher entzündungshemmender Wirkung des Öls.

\section{Erntezeitpunkt entscheidend für die Zusammensetzung}

Eine italienische Arbeitsgruppe unterzog die unverseifbare Fraktion aus geschredderten ganzen Oliven verschiedener Reifestadien einer gaschromatografischen Analyse. Der Gesamtgehalt der unverseifbaren Fraktion zeigte über die verschiedenen Erntezeitpunkte der Oliven (20-32 Wochen nach der Blüte) ein Maximum in der 22. Woche. Verglichen mit dem Gehalt in kommerziell erhältlichem Olivenöl „extra vergine“ von etwa 0,5\% (w/w) unverseifbarer Inhaltsstoffe zeigte die Charge aus der 22. Woche nach Blüte einen etwa 5-fach höheren Anteil unverseifbarer Inhaltsstoffe (ca. 2,5\% w/w). Für die anschließenden qualitativen und semiquantitativen Analysen mittels GC/ MS verwendeten die Forscher Chargen aus den Erntewochen 22, 25 und 32. Das Spektrum der Inhaltsstoffe umfasste zu allen Reifezeitpunkten Squalen, $\beta$-Sitosterol, $\alpha$-Tocopherol, Lanosterol, Stigma-

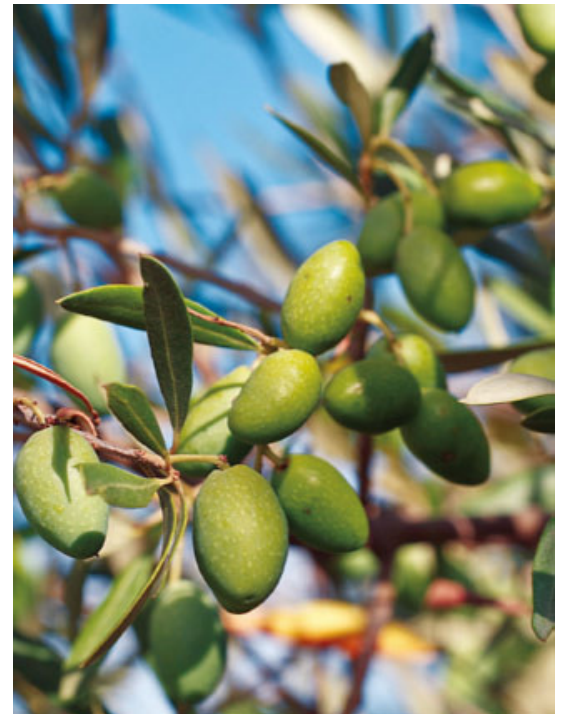

Ausnahmsweise steht hier nicht das Öl im Mittelpunkt, sondern dessen unverseifbare Fraktion. (C) INRA/Jean Weber

sterol. Allerdings konnten nur in der Charge aus Woche 22 folgende weitere Verbindungen nachgewiesen werden: $\alpha$-Amyrin, $\beta$-Amyrin, Stigmastan-3,5-dien, Obtusifoliol, Cycloartanolacetat, Cholesta-4,6-dien3-on, 9,19-Ciclolanost-7-en-3-ol und Cholest-4-en-3-on. Einige davon gelten als mutmaßliche Vorstufen im Sterol-Syntheseweg. Diese Ergebnisse machen die deutlichen qualitativen Veränderungen der bioaktiven Komponenten im Verlauf des Reifeprozesses sichtbar. Eine ähnliche Variabilität zeigte sich auch semiquantitativ.

\section{Klinische Pilotstudie an \\ Osteoarthrose-Patienten}

In einer sehr kleinen Pilotstudie sollten 5 Patienten mit Hand- oder Kniearthrose über den Zeitraum von 3 Wochen 3-mal täglich eine Creme auftragen, die 5\% der bioaktiven unverseifbaren Verbindungen zum Erntezeitpunkt 22. Woche enthielt. $\mathrm{Zu}$ Studienbeginn und jeweils nach einer Woche bis Studienende sollten die Probanden anhand einer visuellen Analogskala (VAS) ihre Gelenksymptomatik beurteilen. Dazu gehörten der Schmerz, die Rötung, die Erwärmung, Ödeme, Beweglichkeit und Gelenkdeformation. Insbesondere die Gelenkschmerzen verringerten sich im Verlauf der Anwendung deutlich von durchschnittlich 8,0 auf 2,2 auf der VAS. Ebenfalls signifikant gingen Röte, Erwärmung, Ödeme und die eingeschränkte Beweglichkeit der Gelenke zurück. Wie zu erwarten war, hatte die Creme keinen Einfluss auf die Gelenkdeformation. Insbesondere der Gehalt an Phytosterolen, $\alpha$ - und $\beta$-Amyrin, $\alpha$-Tocopherol und Squalen wird von den Autoren der Studie für die pharmakologische Wirkung verantwortlich gemacht. Denn diese Verbindungen wirken auf verschiedene Entzündungsparameter (u.a. Prostaglandine, Interleukine und Cyclooxygenase) ein oder fangen freie Radikale ab.

\section{Fazit}

Eine Creme mit 5\% der unverseifbaren Olivenöl-Inhaltsstoffe aus der 22-Reifewochen-Charge reduzierte in einer Pilotstudie bei Arthrosepatienten signifikant mehrere subjektiv empfundene Entzündungssymptome.

\section{Ulrike Andres, Ginsheim}

\section{Literatur}

Gelmini F, Ruscica M, Macchi C et al. Unsaponifiable fraction of unripe fruits of Olea europea: an interesting source of anti-inflammatory constituents. Planta Med 2016; 82: 273-278 